\title{
Abrupt Transition in Quasiparticle Dynamics at Optimal Doping in a Cuprate Superconductor System
}

\author{
N. Gedik, ${ }^{*}$ M. Langner, and J. Orenstein \\ Physics Department, University of California, Berkeley, Berkeley, California, USA \\ and Materials Science Division, Lawrence Berkeley National Laboratory, Berkeley, California 94720, USA
}

S. Ono, Yasushi Abe, ${ }^{\dagger}$ and Yoichi Ando

Central Research Institute of Electric Power Industry, Komae, Tokyo 201-8511, Japan

(Received 15 March 2005; published 9 September 2005; corrected 22 September 2005)

\begin{abstract}
We report time-resolved measurements of the photoinduced change in reflectivity, $\Delta R$, in the $\mathrm{Bi}_{2} \mathrm{Sr}_{2} \mathrm{Ca}_{1-y} \mathrm{Dy}_{y} \mathrm{Cu}_{2} \mathrm{O}_{8+\delta}$ (BSCCO) system of cuprate superconductors as a function of hole concentration. We find that the kinetics of quasiparticle decay and the sign of $\Delta R$ both change abruptly where the superconducting transition temperature $T_{c}$ is maximal. These coincident changes suggest that a sharp transition in quasiparticle dynamics takes place precisely at optimal doping in the BSCCO system.
\end{abstract}

DOI: 10.1103/PhysRevLett.95.117005

PACS numbers: $74.25 . \mathrm{Gz}, 78.47 .+\mathrm{p}$

Pump and probe methods in optical spectroscopy have opened a new window on the properties of quasiparticles in cuprate superconductors and other highly correlated electron systems [1]. In experiments based on these methods, ultrashort pump pulses inject quasiparticles at densities that are continuously variable from well above to well below the thermal equilibrium level. Time-delayed probe pulses measure changes in the reflectivity or transmissivity that result from the presence of nonequilibrium quasiparticles, providing information about their recombination rates, transport, and optical properties. These studies have been carried out extensively in the cuprate superconductors, yielding a rich, complex, yet poorly understood array of experimental observations. One of the central observations, and possibly the most puzzling, has been the behavior of the quasiparticle recombination rate, $\gamma$, as a function of temperature, $T$, and photoinjected density, $\Delta n_{\mathrm{ph}}$. Two classes of behavior are found: in class (1) $\gamma$ appears to vanish as $T$ [2-6] and $\Delta n_{\mathrm{ph}}$ $[2,5,7,8]$ tend to zero, while in class (2) $\gamma$ remains essentially constant with decreasing $T$ [9-11] and $\Delta n_{\mathrm{ph}}$ [12]. Another, seemingly distinct, puzzle concerns the sign of the photoinduced change in sample reflectivity, $\Delta R$, which can be either positive or negative [2,9,13-15].

Here we report measurements of $\Delta R$ and $\gamma$ in the $\mathrm{Bi}_{2} \mathrm{Sr}_{2} \mathrm{Ca}_{1-y} \mathrm{Dy}_{y} \mathrm{Cu}_{2} \mathrm{O}_{8+\delta}$ (BSCCO) system of cuprate superconductors as a function of hole concentration, $x$, that considerably clarify the conditions under which these behaviors appear. As discussed below, the key to successfully exploring the BSCCO system was to eliminate the effects of laser-induced heating. Once this is accomplished, we find that the dynamics change from class (1) to (2) at exactly $x_{m}$, the value for which the superconducting transition temperature $T_{c}$ is maximal. Moreover, we find that the sign of $\Delta R$ reverses at $x_{m}$ as well. These coincident changes suggest that an abrupt transition in quasiparticle dynamics takes place precisely at optimal doping in the BSCCO system.

Time-resolved optical spectroscopy was performed using pump and probe pulses of photon energy $1.5 \mathrm{eV}$ and duration 80 fs from a mode-locked Ti:Sapphire oscillator. Because the BSCCO crystals are optically thick at the laser wavelength of $820 \mathrm{~nm}$, the changes in optical response were probed by measuring the reflected probe power. Figure 1 is a plot of the initial reflectivity change, $\Delta R$, normalized to the reflectivity $R$, as a function of the energy per area, $\Phi_{L}$, deposited by each pump pulse. The underdoped $\left(T_{c}=71 \mathrm{~K}\right)$ sample was thermally anchored to a $\mathrm{Cu}$ plate maintained at $5 \mathrm{~K}$. The $\Delta R$ values plotted as open symbols were measured using the full repetition rate of the oscillator, which is $90 \mathrm{MHz}$. At this repetition rate, the slope of $\Delta R / R$ vs laser intensity changes abruptly when $\Phi_{L}$ reaches $0.8 \mu \mathrm{J} / \mathrm{cm}^{2}$. This effect was not observed in

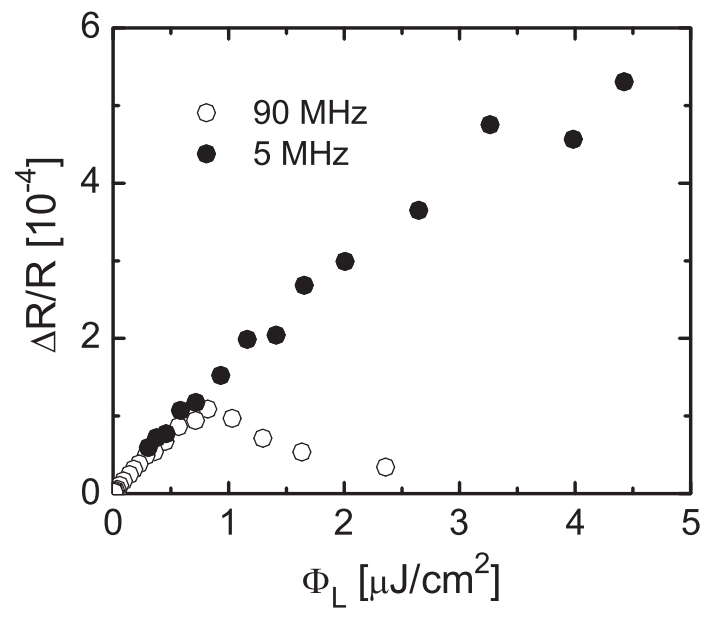

FIG. 1. Fractional change in reflectivity at $1.5 \mathrm{eV}$ as a function of pump energy/(area-pulse), for two values of the pump pulse repetition rate. 
studies of underdoped $\mathrm{YBa}_{2} \mathrm{Cu}_{3} \mathrm{O}_{6.5}$ crystals with similar values of $T_{c}$ and the same conditions of photoexcitation $[5,8]$.

The decrease in $\Delta R / R$ with increasing $\Phi_{L}$ occurs when photoexcitation begins to drive the surface of the sample into the normal state. The origin of this effect is the steady state increase in the surface temperature of the sample due to laser-induced heating. The effects of surface heating are more severe than in the $\mathrm{YBa}_{2} \mathrm{Cu}_{3} \mathrm{O}_{\{7-\delta\}}$ system because $c$-axis thermal conductivity of $\mathrm{BSCCO}$ is much smaller [16]. To overcome the laser-heating problem, we inserted an acousto-optic pulse picker at the laser output. The solid symbols in Fig. 1 indicate values of $\Delta R / R$ obtained when the pulse picker reduces the $90 \mathrm{MHz}$ pulse-repetition rate of the laser to $5 \mathrm{MHz}$ (at the same time the diameter of the illuminated area on the sample was reduced from 75 to $30 \mu$ ). When the average power is thus reduced (by a factor of $\sim 100$ ) the discontinuity in the slope of $\Delta R$ vs $\Phi_{L}$ disappears and the growth of $\Delta R / R$ with $\Phi_{L}$ is essentially linear. Further reduction of the repetition rate to $2.5 \mathrm{MHz}$ produced no further changes in the either the amplitude or the subsequent decay of $\Delta R / R$, indicating that effects of laser heating are negligible at this power level.

Eliminating problems associated with laser heating makes it possible to investigate the nonequilibrium state of highly anisotropic cuprate superconductors at low temperature. In this work we studied eight BSCCO crystals whose $T_{c}$ 's range from $42 \mathrm{~K}$ (underdoped) to $77 \mathrm{~K}$ (overdoped). To control hole concentration over a wide range, we combined both oxygen tuning and Dy-doping. We achieved the target $T_{c}$ 's by floating-zone growth of three different kinds of single crystals with the following Bi:Sr:Ca:Dy ratios: (a) 2.10:1.91:1.03:0,

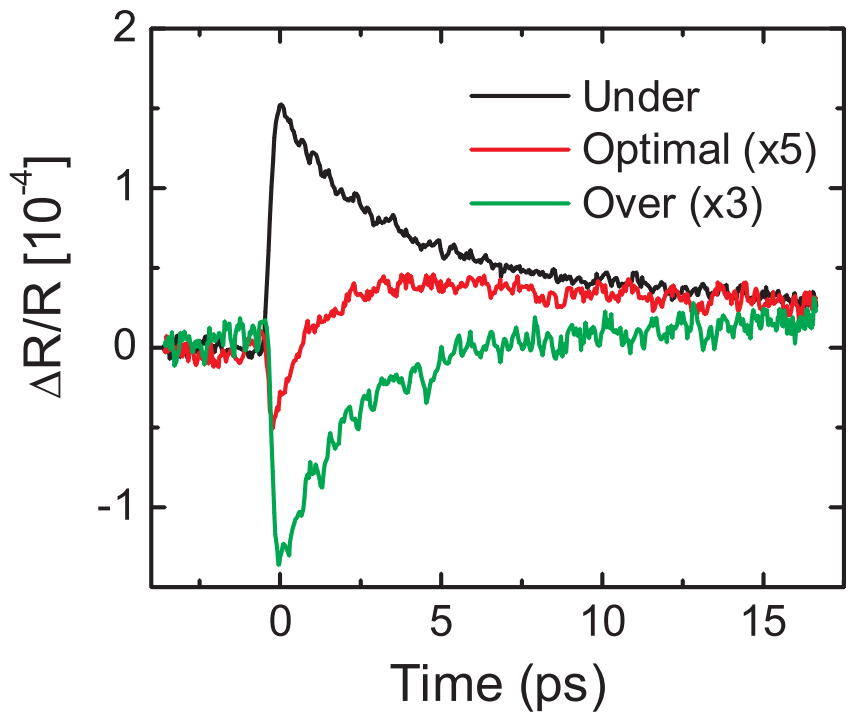

FIG. 2 (color). Fractional change in reflectivity as a function of time following pulsed photoexcitation, comparing the response of underdoped, optimal, and overdoped samples. (b) 2.11:1.88:0.79:0.23, and (c) 2.13:1.82:0.70:0.36. The crystals were subsequently annealed for 2 to 14 days, depending on the annealing temperature, in air or an argon + oxygen environment.

We now describe the evolution of the photoinduced $\Delta R$ with hole concentration. Figure 2 presents an overview of the changes, showing $\Delta R$ (at $\Phi_{L}=0.9 \mu \mathrm{J} / \mathrm{cm}^{2}$ ) as a function of time for three representative samples: underdoped $\left(T_{c}=71 \mathrm{~K}\right)$, optimally doped $\left(T_{c}=94.5 \mathrm{~K}\right)$, and overdoped $\left(T_{c}=77 \mathrm{~K}\right) . \Delta R$ changes from positive for the underdoped sample to negative for the overdoped sample. In the sample with $T_{c}=94.5 \mathrm{~K}, \Delta R$ is a superposition of signals of both sign, indicating that the crossover takes place precisely at optimal doping.

Figures 3(a) and 3(b) illustrate the crossover in kinetics that takes place at the same hole concentration at which $\Delta R$ changes sign. The top panels show $\Delta R(t) / R$ at different values of $\Phi_{L}$, for the same under and overdoped samples as in Fig. 2. For each set of curves, a plot of the initial decay rate, $\gamma(0)$, vs $\Phi_{L}$ appears below. In underdoped samples the decay rate increases linearly with $\Phi_{L}$. We emphasize that $\Delta R(t)$ is independent of the pulse-repetition rate in this regime, proving that the increase in decay rate is not an artifact of laser heating combined with a strongly $T$-dependent $\gamma(0)$. The adjacent panels illustrate that the kinetics of photoexcitations in overdoped samples are substantially different - the decay rate of the excited state remains large as $\Phi_{L}$ is lowered.

The abruptness of the change in sign and decay rate of $\Delta R$ with hole concentration $x$ is shown in Figs. 4(a) and
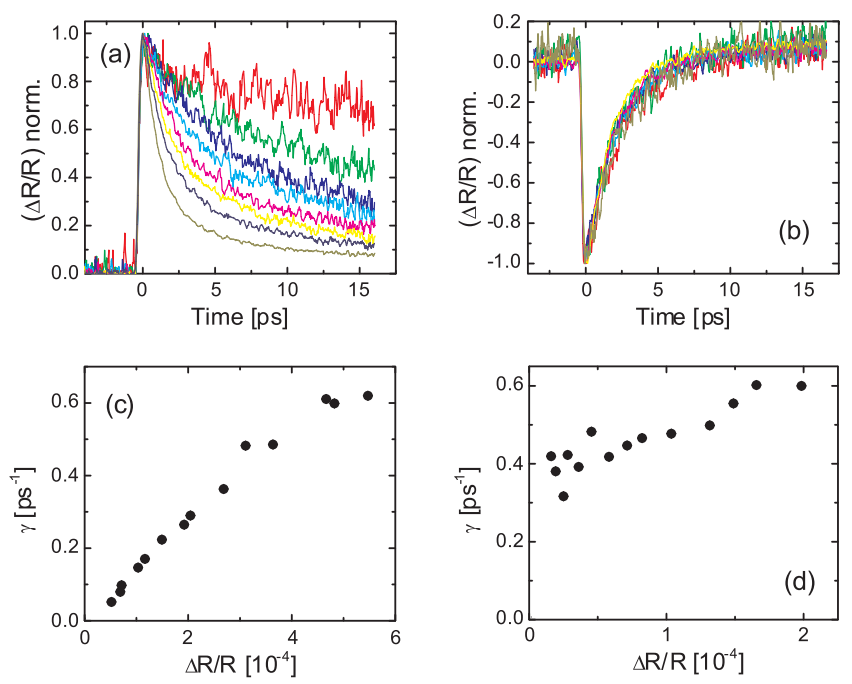

FIG. 3 (color). Top panels: Fractional change in reflectivity as a function of time for an (a) underdoped $\left(T_{c}=71 \mathrm{~K}\right)$ and (b) overdoped sample $\left(T_{c}=77 \mathrm{~K}\right)$, for several values of the pump fluence in the range from $0.1 \mu \mathrm{J} / \mathrm{cm}^{2}$ (red curve) to $1.0 \mu \mathrm{J} / \mathrm{cm}^{2}$ (dark green curve). The plots are scaled to have the same value at $t=0$, illustrating that the decay rate depends on fluence in underdoped samples but not in overdoped samples. Bottom panels (c) and (d) Initial decay rate as a function of initial $\Delta R / R$ for the curves directly above. 
4(b). Figure 4(a) is a plot of $\Delta R$ vs $x$ measured at 0.2 ps after arrival of the pump pulse. The values of $x$ were obtained from the $T_{c}$ 's using the empirical formula given in Ref. [17]. Figure 4(b) shows the initial decay rate of $\Delta R$ for the same set of samples, measured at $\Phi_{L}=$ $0.3 \mu \mathrm{J} / \mathrm{cm}^{2}$. At this relatively low $\Phi_{L}$, the lifetime changes by a factor $\sim 50$ as $x$ varies from just below to just above $x_{m}$. In the following, we discuss the origin of the two transitions, starting with the sign of $\Delta R$.

To analyze the sign change of $\Delta R$ we consider the change in the real part of the dielectric function, $\Delta \epsilon_{1}$, that accompanies photoexcitation of the superconductor. Photoexcitation transfers spectral weight from the condensate $\delta$ function at $\omega=0$ to higher frequency $[10,18]$. The sign of the resulting $\Delta \epsilon_{1}$ depends on the relative ordering of the probe frequency, $\omega_{0}$, and the maximum frequency, $\omega_{\max }$, where the condensate spectral weight reappears. When $\omega_{0} \gg \omega_{\max }$, the Kramers-Kronig relations imply that $\Delta \epsilon_{1} \simeq-\left(8 / \omega_{0}^{4}\right) \int d \omega \Delta \sigma_{1}(\omega) \omega^{2}$, where $\Delta \sigma_{1}$ is spectrum of the conductivity that was removed from the $\delta$ function [5]. This limit applies to BCS superconductors, where all the condensate spectral weight shifts to low frequencies, on the scale of the quasiparticle scattering rate, $1 / \tau$. As $\Delta \sigma_{1}$ is positive, $\Delta \epsilon_{1}$ must be negative, implying that $\Delta R<0$ at the probe energy of $1.5 \mathrm{eV}$ [19].

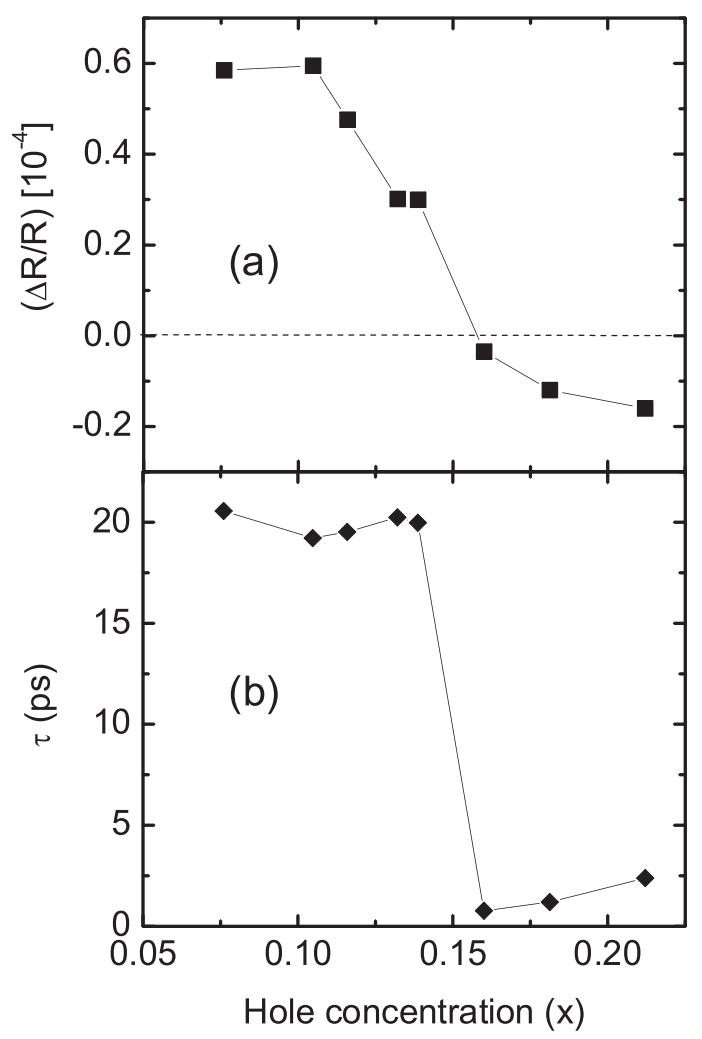

FIG. 4. (a) Fractional change in reflectivity and (b) initial lifetime as a function of hole concentration. All data was measured at pump laser fluence $0.3 \mu \mathrm{J} / \mathrm{cm}^{2}$. The collapse of the quasiparticle lifetime and sign change of $\Delta R$ both occur at optimal doping.
Thus the spectral weight shifts expected for a BCS superconductor can account for the photoinduced response in overdoped, but not underdoped BSCCO [20,21].

To obtain $\Delta \epsilon_{1}>0$ of the opposite sign a fraction of the spectral weight removed from the condensate must shift to $\omega \gg \omega_{0}$, or become broad on the scale of $\omega_{0}$. In other words, some spectral weight must be distributed on the scale of the electronic bandwidth, rather than the frequency scale of $1 / \tau$. In this case $\Delta \epsilon_{1}$ acquires a positive contribution, given approximately by $8 A / \omega_{0}^{2}$, where $A$ is the spectral weight removed from the condensate that is shifted to high frequencies [22]. The magnitude of $A$ that we observe is quite comparable to the value recently found to accompany thermal, rather than photoinduced depletion of the condensate [22]. In the interpretation of the thermal experiments, $A$ is related to a change in electron kinetic energy, $\langle T\rangle$, through the relation, $A \equiv \int \sigma_{1} d \omega=\left(\pi e^{2} / 2 \hbar^{2} d\right) \times$ $\langle-T\rangle$ [23]. Using the preceding formula, the largest $\Delta R$ that we obtain, $\sim 4 \times 10^{-4}$, corresponds to an increase in kinetic energy due to condensate depletion of $\sim 3 \mathrm{meV}$.

We next discuss the discontinuous change in decay rate that takes place at the same hole concentration at which $\Delta R$ changes sign. The sudden change in rate at $x_{m}$ marks the transition from $\gamma(0) \propto \Phi_{L}$ on the underdoped side of the phase diagram to $\gamma(0)$ independent of $\Phi_{L}$ on the overdoped side. For underdoped samples, the decay rate is a linear function of the density of photoinduced excitations. Such "second-order kinetics" occurs when individual, isolated excitations are stable (or metastable) and the rate of decay is limited by the frequency of two-quasiparticle encounters. We note that second-order kinetics are not observed in low- $T_{c}$, BCS-like superconductors because phonons emitted during recombination rapidly regenerate quasiparticle pairs [24]. Decay of the nonequilibrium state is then a first-order process limited by the lifetime of gap-energy phonons. However, the transition to a $\Phi_{L}$-independent decay rate at optimal doping cannot be ascribed to the onset of a phonon-bottleneck because $\gamma(0)$ for $x>x_{m}$ is larger than the pair-recombination rate. Instead, it must be that the excitations that were metastable on the underdoped side of the phase diagram become unstable on the overdoped side. Below, we discuss the nature of these excitations and potential explanations for the metastableunstable transition.

The linear dependence of the magnitude of $\Delta R$ on $\Phi_{L}$ suggests that the excitations that give rise to $\Delta R$ are antinodal quasiparticles. The spectral weight removed from the condensate by nodal quasiparticles would be proportional to $\Delta n_{\mathrm{ph}}^{1 / 2}$ and $\Delta R$ would be proportional to $\Phi_{L}^{1 / 3}$ [25]. The observation of second-order kinetics suggests that antinodal quasiparticles are metastable for $x<x_{m}$. The rapid, $\Delta n_{\mathrm{ph}}$-independent decay of $\Delta R$ that appears for $x>x_{m}$ may indicate a sudden change in the dynamics of antinodal quasiparticles at $x_{m}$. This change is consistent with angleresolved photoemission spectroscopy investigations [26] of the quasiparticle self-energy as a function of $x$, particu- 
larly the recent emphasis on a "dichotomy" between nodal and antinodal excitations [27-29]. The dichotomy exists on the underdoped side, where excitations near the node are coherent quasiparticles and antinodal excitations are incoherent. With increasing $x$ the quasiparticle coherence extends further from the node and appears to encompass the entire Fermi surface in overdoped samples. We speculate that the metastability for $x<x_{m}$ exists because incoherent antinodal excitations cannot readily convert to coherent nodal excitations. When the entire Fermi surface becomes coherent the rapid conversion of antinodal to nodal quasiparticle becomes allowed.

Further evidence that the excitations in underdoped samples are incoherent comes from the second-order recombination coefficient, $\beta$, defined such that $\gamma(0)=$ $\beta \Delta n_{\mathrm{ph}}$ [24]. To estimate $\beta$ we need to know the number of quasiparticles created per photon absorbed, $n_{0}$. If, as we discussed above, the photoinduced quasiparticles are primarily antinodal, then $n_{0} \leq \hbar \omega_{0} / \Delta$, where $\Delta$ is the antinodal creation energy of $\sim 35 \mathrm{meV}$. Using this assumption, we obtain a lower bound for $\beta$ of $\approx 0.1 \mathrm{~cm}^{2} / \mathrm{s}$, for all the underdoped samples measured in this study. If a particle is incoherent, its bandlike motion is frustrated and the rate of recombination can become limited by the time needed to diffuse the average distance between them. For a 2D "diffusion-limited reaction" process $\beta \simeq D$. It is therefore interesting to compare $\beta$ with the quantum diffusion of a fermion in $2 \mathrm{D}$. At the localization limit $k_{F} l=1, D=$ $1 / h N_{F}$, or $\hbar / 2 m^{*}$, where $N_{F}$ and $m^{*}$ are the Fermi level density of states and effective mass, respectively. If we take $m^{*}=3 m$ as suggested from optical measurements [30], then $D=0.15 \mathrm{~cm}^{2} / \mathrm{s}$, which is remarkably close to the experimental estimate for $\beta$.

In conclusion, we have observed an abrupt change in the quasiparticle decay rate and the sign of the photoinduced $\Delta R$ in BSCCO superconductors precisely at optimal doping. On the underdoped side, the photoinjected quasiparticles appear to propagate incoherently and cause some condensate spectral weight to shift to very high frequencies. The sign of spectral weight shift is consistent with recent suggestions of a kinetic-energy driven transition to the superconducting state [22]. The change in the sign of $\Delta R$ at $x_{m}$ suggests that the reduction in kinetic energy at the onset of superconductivity is primarily a feature of the underdoped regime. The sudden change in quasiparticle dynamics at $x_{m}$ may signal the onset of antinodal quasiparticle coherence in optimal and overdoped samples.

This work was supported by DOE-DE-AC0376 SF00098.

*Present address: Laboratory for Molecular Sciences, Arthur Amos Noyes Laboratory of Chemical Physics, California Institute of Technology, Pasadena, CA 91125 Electronic address: gedik@caltech.edu

†Present address: CERC, AIST, Tsukuba 205-8562, Japan
[1] R. D. Averitt and A. J. Taylor, J. Phys. Condens. Matter 14, R1357 (2002)

[2] P. Gay, D. C. Smith, C. J. Stevens, C. Chen, G. Yang, S. J. Abell, D.Z. Wang, J. H. Wang, Z. F. Ren, and J. F. Ryan, J. Low Temp. Phys. 117, 1025 (1999).

[3] D. C. Smith, P. Gay, C. J. Stevens, D. Z. Wang, J. H. Wang, Z. F. Ren, and J.F. Ryan, J. Low Temp. Phys. 117, 1059 (1999).

[4] J. Demsar, R. Hudej, J. Karpinski, V. V. Kabanov, and D. Mihailovic, Phys. Rev. B 63, 054519 (2001).

[5] G. P. Segre, N. Gedik, J. Orenstein, D. A. Bonn, R. Liang, and W. N. Hardy, Phys. Rev. Lett. 88, 137001 (2002).

[6] M. L. Schneider et al., Europhys. Lett. 60, 460 (2002).

[7] N. Gedik, J. Orenstein, R. X. Liang, D. A. Bonn, and W. N. Hardy, Science 300, 1410 (2003).

[8] N. Gedik, P. Blake, R. C. Spitzer, J. Orenstein, R. Liang, D. A. Bonn, and W. N. Hardy, Phys. Rev. B 70, 014504 (2004).

[9] S. G. Han, Z. V. Vardeny, K. S. Wong, O. G. Symko, and G. Koren, Phys. Rev. Lett. 65, 2708 (1990).

[10] R. D. Averitt, G. Rodriguez, A. I. Lobad, J. L. W. Siders, S. A. Trugman, and A.J. Taylor, Phys. Rev. B 63, 140502(R) (2001).

[11] J. Demsar, B. Podobnik, V. V. Kabanov, T. Wolf, and D. Mihailovic, Phys. Rev. Lett. 82, 4918 (1999).

[12] W. Albrecht, T. Kruse, K. Leo, and H. Kurz, Appl. Phys. A 57, 203 (1993).

[13] G. L. Eesley, J. Heremans, M. S. Meyer, G. L. Doll, and S. H. Liou, Phys. Rev. Lett. 65, 3445 (1990).

[14] D. H. Reitze, A. M. Weiner, A. Inam, and S. Etemad, Phys. Rev. B 46, R14309 (1992).

[15] D. Dvorsek, V. V. Kabanov, J. Demsar, S. M. Kazakov, J. Karpinski, and D. Mihailovic, Phys. Rev. B 66, 020510(R) (2002).

[16] M.F. Crommie and A. Zettl, Phys. Rev. B 43, 408 (1991).

[17] M. R. Presland, J. L. Tallon, R. G. Buckley, R. S. Liu, and N. E. Flower, Physica (Amsterdam) 176C, 95 (1991).

[18] M. A. Carnahan, R. A. Kaindl, J. Orenstein, D. S. Chemla, S. Oh, and J.N. Eckstein, Physica (Amsterdam) 408C410C, 729 (2004).

[19] J. Hwang, T. Timusk, and G. D. Gu, Nature (London) 427, 714 (2004).

[20] J. Demsar, R. D. Averitt, V. V. Kabanov, and D. Mihailovic, Phys. Rev. Lett. 91, 169701 (2003).

[21] N. Gedik, J. Orenstein, R. Liang, D. A. Bonn, and W. N. Hardy, Phys. Rev. Lett. 91, 169702 (2003).

[22] H. J. A. Molegraaf, C. Presura, D. van der Marel, P. H. Kes, and M. Li, Science 295, 2239 (2002).

[23] R. Kubo, J. Phys. Soc. Jpn. 12, 570 (1957).

[24] A. Rothwarf and B. N. Taylor, Phys. Rev. Lett. 19, 27 (1967).

[25] J. P. Carbotte and E. Schachinger, Phys. Rev. B 70, 014517 (2004).

[26] A. Damascelli, Z. Hussain, and Z.X. Shen, Rev. Mod. Phys. 75, 473 (2003).

[27] T. Yoshida et al., Phys. Rev. Lett. 91, 027001 (2003).

[28] X. J. Zhou et al., Phys. Rev. Lett. 92, 187001 (2004).

[29] K. M. Shen et al., Science 307, 901 (2005).

[30] Y.-S. Lee, K. Segawa, Y. Ando, and D. N. Basov, Phys. Rev. B 70, 014518 (2004). 